\title{
Dissolution/In Vitro Release Testing of Special Dosage Forms*
}

\author{
Vinod P. Shah,1 Martin Siewert,2 \\ Jennifer Dressman, 3 Helga Moeller, 4 Cynthia K. Brown 5
}

\section{Contributors}

Jean-Marc Aiache, ${ }^{6}$ Cynthia K. Brown, ${ }^{5}$ Jennifer Dressman ${ }^{3}$, Ruzica Djerki, ${ }^{7}$ Kirsti Gjellan ${ }^{8}$, Vivian Gray, ${ }_{1}^{9}$ J ohannes Kraemer, ${ }^{10}$ Henning Kristensen, ${ }^{11}$ Christian Leuner, ${ }^{3}$ Petra Loos, ${ }^{2}$ Helga Moeller, ${ }^{4}$ Berndt Mueller, ${ }^{12}$ Saeed Qureshi, ${ }_{13}^{13}$ J oseph Robinson, ${ }^{14}$ Vinod P. Shah, ${ }^{1}$ and Martin Siewert. ${ }^{2}$

*This article is representing the conclusions from several experts and, in particular, is derived from a series of workshopsheld under the auspices of FIP. It is published as a draft position paper to invite comments from the scientific, technical and regulatory community.FIP and AAPS will co-sponsor a third workshop in September 2002 after which, and including comments on this article, a final FIP position paper will be published.

Please send comments to the Chairman of the FIP Dissolution Working Group:

Dr.Martin Siewert

Aventis Pharma AG

Poseidon Bldg.Room 942

D-65926 Frankfurt am Main

Germany

email:martin.siewert@aventis.com

fax:+496930516692

${ }^{1}$ Food and Drug Administration, Rockville,MD

${ }^{2}$ Aventis Pharma, Frankfurt, Germany

3 JW Goethe University, Frankfurt, Germany

4 Zentrallaboratorium Deutscher A potheker, Eschborn, Germany

${ }^{5}$ Quintiles, Inc. Kansas City, MO

${ }^{6}$ Univesite d'Auvergne, Clarmont-Ferrand Cedex, France

7 Novartis Pharma, Switzerland

${ }^{8}$ AstraZeneca, Sodertalje, Sweden

9 V. A. Gray Consulting, Inc., Hockessin,DE

${ }^{10}$ Laboratory and Quality Services, Eschborn, Germany

${ }^{11}$ University of Copenhagen, Denmark

${ }^{12}$ Kiel University, Germany

${ }^{13}$ Health Protection Branch, Canada

${ }^{14}$ University of Wisconsin,USA

\section{Concept of Dissolution/Drug Release Testing}

$\mathrm{n}$ the pharmaceutical industry, dissolution testing is a very important tool in drug development and quality control. Although initially developed for solid oral dosage forms, in recent years, the application of dissolution testing has widened to a variety of "special"dosage forms, such as suspensions, chewable tablets, chewing gums, transdermal patches, semi-solid preparations, suppositories and implants. For drug products administered orally, it is customary to refer to the test as a 'dissolution' test, because the drug dissolves in the test medium. For non-oral dosage forms such as topical and transdermal delivery systems, suppositories and others, the test is preferably referred to as a 'drug release' or 'in vitro release' test procedure. Due to significant differences in formulation design among these special dosage forms, which in turn lead to very different physicochemical characteristics, it is not possible to devise a single test system which could be used to study the drug release properties of each and every one. Rather, different ap paratus, procedures and techniques are employed on a case-by-case basis, which could be dosage form, type of formulation, or even product specific.

However, the general principlesand application of dissolution testsfor solid oral dosage forms should also be applicable to in vitro/drug release tests for special dosage forms. The ultimate goal of these tests is analogous to that for solid oral dosage forms i.e. to use the test for the biopharmaceutical characterization of the drug product, and as atool to assure consistent product quality within a defined set of specification criteria.

Different types of dosage forms and appropriate apparatus used fordrug release testing are discussed below. For several special dosage forms, the methodology is well evolved and specific recommendations can be made for drug release testing, e.g., for suspensions, chewable tablets, suppositories, transdermal patches and semi-solid topical dosage forms (creams, ointments and gels). As for conventional oral dosage forms there may be specific formulations in the above-mentioned categories for which the evolved methods may not be 
applicable. In several other instances, e.g., chewing gums, powders, granules, solid dispersions, microparticles, and implants,more method development and refinement will be required before a final recommendation on standardized drug release methods can be made.For these dosage forms, a brief summary of the state-of-the-art knowledge is provided to guide further development. Due to the different characteristics of the special dosage forms and their sites and modes pf application, it is essential that ap paratus selection, composition of the dissolution medium, agitation (flow rate) and temperature be given ap propriate consideration during evaluation of key method parameters. Instances where a compendial (USP,Ph.Eur.,Ph.Jap.) basket or paddle method is employed for in vitro drug release testing, the experimental test conditions, qualifications and validation steps should bethe same as discussed in the FIP Guidelines on dissolution testing (1).

\section{Dosage Forms for Which a Specific Method Can Be Recommended}

\section{Oral Suspensions}

In general, the rotating paddle method utilizing an aqueous dissolution medium is the recommended method for dissolution testing of suspensions. To obtain representative samples, product preparation should follow a standardized procedure based on shaking or mixing.Sample weight/volume should reflect a typical dose of the product. Method parameters such as sample intro duction and agitation rate should be established on the basis of the viscosity and composition of the suspension matrix. The sample introduction technique must be accurate, precise and reproducible. The agitation rate should facilitate discrimination between batches with different release properties.

For low viscosity suspensions, an accurate dose can be delivered to the bottom of the dissolution vessel using a volumetric pipette. A low agitation rate of $25 \mathrm{rpm}$ is generally recommended for less viscous suspensions (2). For high viscosity samples, the dose may need to be determined by weight with a quantitative sample transfer to the dissolution vessel to ensure accuracy of the sample size introduced. High viscosity suspensions may also require a higher agitation rate such as 50 or $75 \mathrm{rpm}$ to prevent sample mounding at the bottom of the vessel.

\section{Chewable Tablets}

In principle, the test procedure employed for chewable tablets should be the same as that for regular tablets. This concept is based on the possibility that a patient might swallow the dosage form without proper chewing, in which case the drug will still need to be released to ensure the desired pharmacological action (3). Where applicable,test conditions would preferably be the same as used for conventional tablets of the same active pharmaceutical ingredient, but because of the non-disintegrating nature of the dosage form, there may be a necessity to alter test conditions (e.g. increase the agitation rate) and specifications (e.g.increase the test duration).

\section{Transdermal Patches}

Although several apparatus and procedures have been utilized to study in vitro release characteristics of transdermal patches, it is desirable to avoid unnecessary proliferation of dissolution test equipment. Current compendial apparatus include paddle over disk (USP apparatus 5), rotating cylinder (USP apparatus 6 ) and reciprocating disk (USP ap paratus 7). As it has been shown experimentally that a simple paddle over disk procedure using a watch glasspatch-screen sand wich assembly results in almost the same release profile as other, more complicated apparatus for all US marketed transdermal patches (4) the paddle over disk procedure with a watch glass-patch-screen sandwich assembly is considered method of choice.

The configuration of this assembly ensures that the patch is prevented from floating during the entire testing period. Special attention needs to be given to the proper positioning of the patch so that the drug-loaded surface is exposed to the medium. The $\mathrm{pH}$ of the medium ideally should be adjusted to $5-6$, reflecting physiological skin conditions. For the same reason, test temperature is typically set at $32^{\circ} \mathrm{C}$.

\section{Semisolid Dosage Forms}

Semisolid dosage forms include creams, ointments and gels. In vitro drug release from semisolid dosage forms has been extensively investigated using the Franz cell diffusion system (5) with a synthetic membrane and to some extent using the Enhancer cell (6). Comparative studies indicate that both types of ap paratus generate similar data. However, in certain instances, especially with gels, the diffusion cell system appears to have some superiority as results are less dependent on specific formulation/composition details (7).

Depending on the solubility of the drug substance,the receptor medium may need to contain alcohol and/or surfactant.De-aeration is critical to avoid bubble formation 


\section{Special Dosage Forms ... continued}

at the interface with the membrane. As with transdermal products the test temperature is typically set at $32^{\circ} \mathrm{C}$ to reflect the usual skin temperature.

\section{Suppositories}

For hydrophilic suppositories that release the drug by dissolving in the rectal fluids, the basket, paddle, or flow-through cell can all be used in principle.

For lipophilic suppositories that release the drug after melting in the rectal cavity, a modified basket method is recommended. A paddle method with a wired screen and a sinker is also recommended (8). For lipo philic sup positories, a modified flowthrough cell with the specific suppository cell has also been evaluated. Experience with this cell has shown that it may generate highly variable data due to the behavior of the sup pository in the cell,in particularfor formulations containing spreading agents. Therefore,this modified flow through cell with the specific suppository cell cannot be recommended generally for quality control and routine analysis of lipophilic suppositories (9).

\section{Dosage Forms Requiring More Work Before a Method Can Be Recommended}

\section{Chewing Gum}

In the case of chewing gums, the intensity and frequency of shearing forces/activities (i.e. "chewing"action) can have a large influence on drug release rate. The European Pharmacopoeia provides a description of an apparatus designed for testing of chewing gum products (10). However,to date there has been insufficient experience with this apparatus to draw a firm conclusion about its suitability.

\section{Powders, Granules, Solid Solutions and Solid Dispersions}

The flow through apparatus offers specific sample cells for studying drug release from powder and granular dosage forms. However, it is important to note that the dissolution behavior of these dosage forms may beg reatly influenced by their wettability, surface area and particle size distribution. Thus, the in vitro release test results constitute one of a group of physicochemical parameters needed to characterize the product. For powders, especially when exhibiting poor wettability, it may be necessary to add a surfactant to the dissolution medium to obtain reproducible dissolution results. Care should be taken to use a level of surfactant that does not increase the solubility of the drug to the extent where the test is no longer discriminatory.

Solid solutions and dispersions may be housed in seemingly typical oral dosage forms such as capsules and tablets. If this is the case, their in vitro release characteristics can be determined by methods typical for solid oral dosage forms. Since solid solutions and dispersions usually lead to a super saturation of the medium, it is often of interest (especially during product development) to run the in vitro release test somewhat longer so that the potential for precipitation can be appraised.

\section{Parenterals: Implants and Microparticles}

The modified flow-through cell has been used successfully for implantsand microparticles. The compendial flow-through apparatus ismodified with regard to the inner diameter to suit the special properties for testing parenterals, i.e. low volume of acceptor compartment. The flow rate of the medium has to be set very slow.As tests are often run over a long time period (e.g.several weeks) measures have to be taken to compensate against evaporation and to prevent microbial growth in the medium. The composition of the medium should take into consideration the osmolarity, $\mathrm{pH}$ and buffer capacity of the fluids at the site of application, which are usually assumed to resemble that of plasma. However, the main challenges with this type of dosage form are to determine the appropriate duration of the test and the times at which samples are to be drawn in order to characterize the release profile ad equately. The possibility of running the test under accelerated conditions is attractive, but to date there has not been enough experience to make any specific recommendations on this approach.

\section{Formulation Characterization}

In order to characterize the release from the dosage form adequately, it is recognized that a drug release profile should be generated, in which release (dissolution) values are determined as a function of time. This multipoint characterization has been in place for modified release dosage forms for some time and is also recommended for slower dissolving immediate release products. Because many of the dosage forms discussed here are complex in terms of composition and release mechanism,they will also require a multipoint drug release test to characterize release from the drug product in general and to test for possible alterations in the release profile during storage. Multipoint tests may also be needed for batch release testing in order to prove a good batch-to-batch consistency.Typical cases where multipoint tests are likely to be needed include transdermal patches, semisolid preparations, chewing gums, implants, microparticles, solid solutions and solid dispersions. However, in other cases like powders, 
granulates, suspensions, chewable tablets and rapidly releasing suppositories a single point specification may be sufficient for batch-to batch quality control, if properly derived from profiles generated during the development phase of the product.

\section{Experimental Test Conditions}

The experimental test conditions should be discriminating enough ("mild"conditions) to detect manufacturing variables that may affect biopharmaceutical product performance. Test conditions that may not be able to discriminate adequately among products/batches with different in vivo release profiles include those with very high agitation/flow rates, the use of strongly alkaline solutions to dissolve poorly solub le acids, and the use of very high surfactant concentrations to create sink conditions, to name but a few.

As for solid oral dosage forms, development of in vitro release/dissolution tests and specifications for "special"dosage forms should take into account relevant bioavailability data.Ideally, the in vitro/dissolution release test conditions should be selected taking into consideration physiological conditions at the site of application. The complexity of the release mechanism of some special dosage forms and the lack of knowledge ab out the conditions under which release occurs in vivo make it difficult to design physiologically based tests in all cases, but it should be possible to conceive a test that can detect the influence of critical manufacturing variables, differentiate between the different degrees of product performance and to some extent characterize the biopharmaceutical quality of the dosage form.

As the release mechanism and site of application varies dramatically among the special dosage forms, the experimental test conditions have to be tailored according to the conditions at the site of application (e.g.temperature of the test) and the release mechanism (e.g.chewing gums will require different agitation rates than suspensions). Within a given category, it may be necessary to have product-type specific dissolution tests (e.g.separate tests for lipophilic and hydrophilic suppositories), and in some cases for products containing the same drug and administered in the same type of special dosage form, but with a different release mechanism (similar to the range of tests available for theophyllin extended release dosageforms).

Test proceduresfor dissolution testing of solid oral dosage forms, i.e. immediate release and modified release dosage forms, have been significantly refined and standardized over the past quarter century. The methods are well on their way to harmonization. It is anticipated that through further refinement and standardization of in vitro release testing for rnonoral and "special"dosage forms, harmonization of tests for these dosage forms will take considerably less time.

\section{Applications}

A specific value of in vitro dissolution / drug release testing is recognized in its application as a batch-to-batch quality control test and its value in Scale-Up and Post Approval Changes (SUPAC). The SUPAC document (for semisolid dosage forms) defines the levels of changes with respect to component and composition, site of manufacturing, scale of manufacturing and process and equipment changes (11). In vitro drug release is used to assure product sameness und er SUPAC related changes. The same principles can easily be extended to other dosage forms where the product sameness can be assured by profile comparison between pre-change and post-change products using an appropriate in vitro test and profile comparison (12). In addition to this, the drug release / dissolution test can also be used for providing bio-waivers for lower strengths of a product from a given manufacturer, once the higher strength is approved based on appropriate bioavailability/bioequivalence test procedure.

\section{Setting Specifications: Acceptance Criteria/Limits}

The in vitro dissolution / drug release specifications should be primarily based on manufacturing experience and pivotal clinical trial batches or other biobatches. Compared to testing of solid dosage forms in basket and paddle dissolution equipment, far less experience is availab le for many of the special dosage forms with respect to variability of data and, where the newer types of apparatus are used, qualification of the equipment. In general, criteria and specification limits (ranges) may be set similar to the procedure for oral solid dosage forms. However, further experience needs to be gained to better understand the desired level of standardization, and it can be expected that the appropriate ranges and criteria for acceptance of release data of special dosage forms will be different to those for solid oral dosage forms in some instances.

\section{Conclusions}

An appropriate drug release test is required to characterize the drug product and assure batch-tobatch reproducibility for consistent pharmacolog- 


\section{Special Dosage Forms ... continued}

ical/biological activity.Table 1 summarizes different apparatus that have been used/can be used for various types of dosage forms. The in vitro drug release test for some "special"dosage forms such as semi-solid dosage forms and transdermal drug delivery systems has proven to be equally valuable as the dissolution test for solid oral dosage forms. The in vitro drug release test also shows promise to be of value for other dosage forms such as chewable tablets, suspensions and suppositories. For yet other dosage forms such as chewing gums, powders, and parenterals, further method development and refinement will be needed to make the drug release test a generally applicable, robust and valuable tool.

\section{References:}

1. FIP Guidelines for Dissolution Testing of Solid Oral Products,Die Pharmazeutische Industrie 59: 760 - 766 (1997)

Table 1: Apparatus used for Special Dosage Forms

\begin{tabular}{|ll|}
\hline Type of Dosage Form & Release Method \\
\hline Solid Oral Dosage Forms & Basket or Paddle \\
\hline Topicals- Semisolids & Diffusion Cell \\
Transdermals- Patches & Paddle over disk \\
Suppositories & Basket or Paddle \\
Suspensions & Paddle \\
ChewableTablets & Basket or Paddle \\
Chewing Gum & Special apparatus \\
Microparticles & Flow through cell \\
Implants & Flow through cell? \\
\hline
\end{tabular}

and Dissolution Technologies 4:5-14 November (1997).

2. USP 24 / NF 19,<1088> page 2051,2000.

3. FDA Guidance for Industry: Bioavailability and Bioequivalence Studies for orally administered drug productsGeneral Considerations, October 2000.

4. VP Shah, NWTymes and J P Skelly, In vitro release profile of clonidine transdermal therapeutic systems and scopolamine patches, Pharm Res 6:346-351 (1989).

5. VPShah,JSElkins and RL Williams, Evaluation of the test system used for in vitro release of drugs from topical dermatological drug products. Pharm Develop Technology;4:377-385 (1999).

6. HM Fares and $J L Z$ Zatz, Measurement of drug release from topical gels using two types of apparatus. Pharm Tech 19(1):52-58 (1995).

7. $P$ Loos, In vitro release from topical dosage forms - significance of current methods for the development of drug products and for post approval changes, Ph.D.thesis (1998)

8. K Kjellan and C Graffner, Comparative dissolution studies of rectal formulations using the basket, the paddle and the flow-through methods. I.Paracetamol in suppositories and soft gelatin capsules of both hydrophilic and lipophilic types. Acta Pharm Nord:343-354 (1989).

9. KGjellan and C Graffner,Comparative dissolution studies of rectal formulations using the basket, the paddle and the flow-through methods. II.Ibuprofen in suppositories of both hydrophilic and lipophilic types. Int J Pharm 112: 233-240 (1994).

10. European Pharmacopoeia 4th Edition - 2002,General Chapter 2.9.25,"Chewing Gum, Medicated, Drug Release From,"pp 227-228, Directorate for the Quality of Medicines of the Council of Europe, Strasbourg,France, 2001.

11. FDA Guidance for Industry:SUPAC-SS Nonsterile semisolid dosage forms. Scale-Up and Post Approval Changes:Chemistry, manufacturing, and controls; in vitro release testing and in vivo bioequivalence documentation, May 1997.

12. GVan Buskirk et.al.,Scale-up of adhesive transdermal drug delivery systems. Pharm Res 14:848-852 (1997). 